\title{
Geometric Flows and Black Hole Entropy
}

\author{
Joseph Samuel and Sutirtha Roy Chowdhury \\ Raman Research Institute, Bangalore-560 080
}

(Dated: June 4, 2018)

\begin{abstract}
Perelman has given a gradient formulation for the Ricci flow, introducing an "entropy function" which increases monotonically along the flow. We pursue a thermodynamic analogy and apply Ricci flow ideas to general relativity. We investigate whether Perelman's entropy is related to (Bekenstein-Hawking) geometric entropy as familiar from black hole thermodynamics. From a study of the fixed points of the flow we conclude that Perelman entropy is not connected to geometric entropy. However, we notice that there is a very similar flow which does appear to be connected to geometric entropy. The new flow may find applications in black hole physics suggesting for instance, new approaches to the Penrose inequality.
\end{abstract}

PACS numbers: 02.40.-k,04.70.Dy 


\section{INTRODUCTION}

The Ricci flow [1, 2, 3, 4] is an evolution equation for Riemannian geometries that tends to smooth and homogenise them. This flow is a (degenerate) parabolic differential equation, and is very similar to the heat equation. This suggests a thermodynamic analogy in which evolution of a geometry along the Ricci flow is like the approach of a physical system to thermodynamic equilibrium. In this paper, we pursue this analogy. We view the Ricci flow from a thermodynamic perspective and apply Ricci flow techniques to isolated gravitating systems in general relativity (GR). Our motivation is to better understand physical quantities like energy and entropy in GR.

This paper is organised as follows. In section II, we briefly describe Perelman's gradient formulation of the Ricci flow. In section III, we motivate the application of these ideas to general relativity. In section IV, we study fixed points of the Perelman flow and note that the Schwarzschild space is not a fixed point. In section $\mathrm{V}$, we describe a slight modification of the Perelman flow. The fixed points of the new flow turn out to be initial data for the Schwarzschild black hole. Section VI generalises the discussion to include AdS. Section VII is a concluding discussion.

\section{THE RICCI FLOW AND PERELMAN'S GRADIENT FORMULATION}

Let $\left(\Sigma, h_{a b}\right)$ be a Riemannian manifold. ( $a, b$ run over 1,2,3. We restrict our discussion to three dimensional manifolds.) Given an initial metric $h_{a b}$, the Ricci flow describes an evolution equation, which evolves the metric according to its Ricci tensor. The evolution parameter is $\tau$ and the family of metrics on $\Sigma, h_{a b}(\tau)$ satisfies the Ricci flow equation

$$
\frac{\partial h_{a b}}{\partial \tau}=-2 R_{a b}
$$

In the neighborhood of a point $p \in \Sigma$, we can introduce a Riemann normal co-ordinate system and then the form of (1) becomes parabolic $\left(\nabla^{2}\right.$ is the Laplacian in local co-ordinates)

$$
\frac{\partial h_{a b}}{\partial \tau}=\nabla^{2} h_{a b}
$$

and looks like a heat equation for the metric coefficients. However, in a general coordinate system, the PDE (1) is a degenerate parabolic equation, because of its diffeomorphism invariance. 
The Ricci flow first appeared in physics in the renormalisation of $\sigma$ models [1]. It was independently introduced in mathematics by Hamilton, who used the flow to understand the topology of three manifolds. Recently, Perelman has spectacularly used Ricci flow techniques to solve the Poincare conjecture and it is widely believed that these techniques also solve Thurston's geometrisation conjecture. These mathematical applications use the Ricci flow to address questions of three dimensional topology. While Perelman's motivation was topological, the techniques he introduced are tools of geometry and analysis. It seems reasonable to hope that these tools will also find physical applications in general relativity. The present paper is a preliminary step in this direction. We suggest that the flow may be useful in understanding elusive concepts like energy and entropy in general relativity. Both of these quantities are well understood in special relativistic physics. In GR[5], they take on a new global geometric interpretation, respectively the mass at infinity and the area of a horizon.

From a physical point of view, the most striking property of the Ricci flow (which it shares with the heat equation) is its tendency to lose memory of initial conditions. This is very similar to the approach of an isolated physical system to thermal equilibrium. For instance, a thermally insulated circular copper wire of circumference $L$, which initially has a temperature distribution $T(x),(T(0)=T(L), T(x)>0)$ evolves according to the heat equation

$$
\frac{\partial T}{\partial t}=\frac{d^{2} T}{d x^{2}}
$$

(by choice of units, we set the diffusion constant to one) and tends to a constant, losing memory of the details of the intial distribution $T(x)$. The final state is characterised by a single number, the uniform final temperature $T_{f}$ and not an entire function $T(x)$. This "information loss" is very reminiscent of entropy increase and indeed, one can view the heat equation in this light. Consider the functional $S[T(x)]=\int_{0}^{L} d x a(T)$, where $a(T)=$ $-T \log T$. Using the heat equation (3), integrating by parts and dropping a divergence we see that $S[T(x)]$ is monotonic along the flow. $\frac{d S}{d t}=\int d x a^{\prime}(T) d T / d t=\int d x a^{\prime}(T) d^{2} T / d x^{2}=$ $-\int d x a^{\prime \prime}(T)(d T / d x)^{2} \geq 0$ The last inequality follows since $a(T)$ is a convex function $a^{\prime \prime}<0$.

The question we address here is: can we in some sense regard the Ricci flow as representing the increase of entropy and the approach of a gravitating system to its equilibrium maximum entropy state. The question can be split into two parts. 1) Is there a functional of the metric which increases monotonically along the Ricci flow? 2) Does this functional physically 
represent geometric entropy?

One answer to the first question is provided by Zamolodchikov's $\mathrm{C}$ theorem, proved in the context of two dimensional $\sigma$ models. This theorem formalises the intuition that renormalisation is an irreversible process. The flow of coupling constants under renormalisation is such that a function $C\left(g_{1}, g_{2} \ldots\right)$ of the coupling constants of the theory is monotonically increasing along the flow. In $\sigma$ models the role of the coupling constants is played by the metric on the target space and the $\beta$ function is the Ricci tensor[1]. The $\mathrm{C}$ theorem guarantees that the RG flow is irrotational and does not have limit cycles. However, the Zamolodchikov C theorem appears to be a very specific result: it applies only to two dimensional field theories and fails if the target space is noncompact. This does not appear to be a general answer to the question we raised.

Another answer, which is more general and relevant to the present context is provided by Perelman's gradient formulation of the Ricci flow. We briefly describe this formulation. Consider the Ricci flow, supplemented by a diffeomorphism $\mathcal{L}_{\xi} h_{a b}=D_{a} \xi_{b}+D_{b} \xi_{a}$ generated by a vector field $\xi^{a}$, which is itself a gradient $\xi_{a}=D_{a} f$, where $f$ is a scalar function on $\Sigma$.

$$
\frac{d h_{a b}}{d \tau}=-2 R_{a b}+2 D_{a} D_{b} f
$$

Perelman gave a gradient formulation for this flow by considering the following "entropy functional" which depends on the pair $\left(h_{a b}, f\right)$, of tensor fields on $\Sigma$.

$$
\mathcal{F}_{\mathcal{P}}\left(h_{a b}, f\right):=\int_{\Sigma} d^{3} x \sqrt{h}(\exp f)\left[R+(D f)^{2}\right]
$$

(In this paper $f$ is reversed in sign from Perelman's equations.) There is a subtlety in the variation however: the degrees of freedom in the metric can be split up into a conformal factor and a conformal structure $\left[h_{a b}(x)\right]$, where the square brackets signify a conformal class. In the variation of $\mathcal{F}_{\mathcal{P}}$, the conformal structure is freely varied, but the conformal factor is subject to the constraint that the "distorted volume" $\sqrt{h}(\exp f)$ is held fixed. Performing a variation $\delta h_{a b}=\frac{d h_{a b}}{d \tau} \delta \tau$ and using the notation $\frac{d h^{a b}}{d \tau}=h^{a c} h^{b d} \frac{d h_{c d}}{d \tau}$, we find using a standard geometric identity for the variation of $R$,

$$
\frac{d R}{d \tau}=-R_{a b} \frac{d h^{a b}}{d \tau}+D_{a} D_{b} \frac{d h^{a b}}{d \tau}-D^{2}\left(h_{a b} \frac{d h^{a b}}{d \tau}\right)
$$

and after dropping some divergences.

$$
\frac{d \mathcal{F}_{\mathcal{P}}}{d \tau}=\int_{\Sigma} d^{3} x \sqrt{h}(\exp f)\left[-R_{a b}+D_{a} D_{b} f\right] \frac{d h^{a b}}{d \tau}
$$


Thus $\frac{d h_{a b}}{d \tau}$ in (4) is twice the gradient of $\mathcal{F}_{\mathcal{P}}$ subject to the constraint of perserving the distorted volume. It follows that $\mathcal{F}_{\mathcal{P}}$ is non decreasing along the flow

$$
\frac{d \mathcal{F}_{\mathcal{P}}}{d \tau} \geq 0
$$

Hence the use of the word "entropy" for the Perelman functional. The evolution equation for $f$ is not independent, but determined by the constraint on the distorted volume. The reader is referred to Topping [6] for a detailed exposition of Perelman's gradient formulation.

In his paper on "The entropy formula for the Ricci flow and its geometric application", Perelman remarks (section 5.3): "The interplay between statistical physics and (pseudo) Riemannian geometry occurs in the subject of black hole thermodynamics developed by Hawking et al. Unfortunately, this subject is beyond my understanding at the moment". Our objective here is to pursue this remark and see if there is any connection between Perelman entropy and the geometric entropy familiar from black hole thermodynamics. This motivates our application of Ricci flow techniques to GR.

\section{APPLICATION TO GENERAL RELATIVITY}

How does one apply Perelman's ideas to general relativity? The Perelman flow is defined in the space of three dimensional Riemannian metrics, while GR deals with four dimensional Lorentzian ones. A natural approach is to use spatial slices of four dimensional spacetimes in order to define the flow. The induced metric on $\Sigma$ is a three dimensional Riemannian metric. In the initial value formulation of $\mathrm{GR}$, one uses the induced metric on $\Sigma$ and the extrinsic curvature of $\Sigma$ as initial data for the spacetime. In this paper we restrict attention to time symmetric initial data. The extrinsic curvature then vanishes and we need to deal only with $\left(\Sigma, h_{a b}\right)$. Our idea is to apply the Ricci flow to the initial data $h_{a b}$. For time symmetric initial data, the dominant energy condition implies that the scalar curvature $R$ is positive. The Ricci flow has the happy feature that it preserves the positivity of scalar curvature[6]. Thus, it may be used to understand general statements about initial data that rely on such assumptions as positivity of curvature. Examples of such statements are the positive energy theorem [7, 8, 9] and the Penrose inequality [10, 11, 12, 13, 14, 15]. In all these examples Einstein's equations do not play any direct role: they are replaced by energy conditions, which are geometric inequalities (for example, the positivity of scalar curvature). 
In general relativity, energy and entropy are only well defined in situations where the space has an asymptotic region. The total mass of an isolated system (the ADM mass) is defined using a fixed metric at infinity (either flat or AdS). The Bekenstein-Hawking entropy of black holes uses the notion of an horizon and "escape to infinity". It is therefore clear that our $\left(\Sigma, h_{a b}\right)$ must be a non-compact space, unlike the compact situations considered by Perelman in the study of three dimensional topology. Let us assume that $\left(\Sigma, h_{a b}\right)$ has just one end at infinity and that $h_{a b}$ tends to a fixed flat metric $\delta_{a b}$ at infinity [5]. Even in ordinary thermodynamics, entropy is only well defined in stationary (or quasistationary) situations. Since we assumed for simplicity that we deal only with time symmetric situations, we can replace the word 'stationary' in the last sentence by static. Correspondingly, in pursuing the thermodynamic analogy we will restrict our attention to static, four dimensional, Lorentzian, asymptotically flat space times, subject to an Energy condition.

We now address the second question: is the Perelman entropy related to geometric entropy, as familiar from black hole physics? Perelman's entropy is non-decreasing along the flow. However, the property of being monotonic along a flow is a fairly generic one and there may be many functionals which have this property. For instance, in black hole physics, the area theorem asserts that the area of a horizon can only increase with time. But then the same is true for the square of the horizon area. In the example of the copper wire above, replacing $a(T)=-T \log T$ by any other convex function $\left(a^{\prime \prime}<0\right)$ would also lead to a functional which monotonically non-decreasing along the flow. Only the choice $a(T)=\log T$ results in $S[T(x)]$ being proportional to the actual thermodynamic entropy of the wire. While all these "entropy functionals" differ, they share one property in common: they are all maximized at the fixed point of the flow: the final state of uniform temperature.

Rather than look at the entropy function, it is more effective to look at the fixed points of the Perelman flow and ask if these correspond to maximum entropy in the physical sense. This motivates a study of the fixed points of the Perelman flow. These fixed points are referred to as 'Ricci solitons'.

The stationary spacetime which maximises its geometric entropy for a fixed energy[16], is the static, spherically symmetric Schwarzschild black hole[17]. Black holes have enormously more entropy than a star of the same mass (some $10^{20}$ times more for a solar mass). If Perelman's entropy were related to geometric entropy, we would expect the Schwarzschild space to be a fixed point of the Perelman flow. This expectation can be tested by a direct 
calculation which is presented in the next section.

\section{RICCI SOLITONS: FIXED POINTS OF THE PERELMAN FLOW:}

The end points of the Perelman flow are called Ricci solitons and are characterised by a vanishing of the RHS of (4). A Ricci soliton consists of a pair $\left(h_{a b}, f\right)$ subject to the equations:

$$
R_{a b}=D_{a} D_{b} f
$$

We wish to check if the Schwarzschild space solves these equations for some choice of $f$. We can assume the spherically symmetric form

$$
d s^{2}=a(r) d r^{2}+r^{2}\left(d \theta^{2}+\sin \theta^{2} d \phi^{2}\right)
$$

for $h_{a b}$ and suppose that $f$ depends only on $r$. In order to preserve this gauge (10) we will need to supplement the RHS of (9) with an additional diffeomorphism generated by a radial vector field $U(r) \frac{\partial}{\partial r}$. Since this vector field is obviously a gradient of a scalar function, we can absorb it in a redefinition of $f$.

The independent fixed point (9) equations are $D^{2} f=R$ and $R_{\theta \theta}=(D D f)_{\theta \theta}$. These are two equations for the pair $(a(r), f(r))$. We can use the second of these equations to eliminate $f(r)$ in favour of $a(r)$ and arrive at the autonomous differential equation for $a(r)$.

$$
\frac{d^{2} a}{d r^{2}}=\frac{3\left(a^{\prime}\right)^{2}}{2 a}+\frac{2 a^{2}-2 a+r a^{\prime}(1-a)}{r^{2}}
$$

It is easily seen that (apart from the trivial case $M=0$ ) the Schwarzschild space, which has

$a(r)=(1-2 M / r)^{-1}$ does not satisfy (11). Thus Schwarzschild is not a fixed point of the flow no matter what choice is made for $f$. This shows that fixed points of the Perelman flow do not maximise geometric entropy as familiar from black hole physics. Flowing the Schwarzschild space using the Perelman flow would lead to a further increase of Perelman's entropy. We conclude that Perelman's entropy function is not connected to Bekenstein-Hawking entropy. However, as we argued earlier, many aspects of the Ricci flow are strongly reminiscent of thermodynamics. Are there other similar flows in the space of metrics which do describe the thermodynamics of geometry? This question is addressed in the next section. 


\section{THE MODIFIED RICCI FLOW AND ITS FIXED POINT}

Let us consider how one can modify the Ricci flow to make connections with black hole entropy. We would like to have a geometric differential equation, (i.e one constructed from tensor fields) of a parabolic nature similar to the forward heat equation. The right hand side must contain no more than second derivatives of the basic fields $\left(h_{a b}, f\right)$. Choosing the simplest possibility consistent with these requirements, we define the modified Ricci flow (MRF) by the equation

$$
\frac{\partial h_{a b}}{\partial \tau}=-2 f R_{a b}+2 D_{a} D_{b} f
$$

This differs from Perelman's flow (4) only in the presence of $f$ in the first term. $f$ can be interpreted as an effective space dependent diffusion constant. It describes a forward type heat equation only in regions where $f$ is positive and bounded away from zero. In these regions we would expect the flow to have the same behaviour as Perelman's flow, smoothing out inhomogeneities and losing memory of initial conditions. Let us supplement the Ricci flow with a forward type evolution equation for $f$ :

$$
\frac{\partial f}{\partial \tau}=D^{2} f
$$

The pair $\left(h_{a b}, f\right)$ evolved according to (1213) constitute the modified Ricci flow [22]. Does the MRF have any connection with geometric entropy?

As we mentioned before, the most efficient way to investigate this question is to study the fixed points of the flow, characterised by

$$
f R_{a b}=D_{a} D_{b} f
$$

where $f$ satisfies

$$
D^{2} f=0
$$

It is easily seen that with

$$
f=(1-2 M / r)^{1 / 2},
$$

the Schwarzschild exterior space

$$
d s^{2}=(1-2 M / r)^{-1} d r^{2}+r^{2}\left(d \theta^{2}+\sin \theta^{2} d \phi^{2}\right)
$$

$(r>2 M)$ is a fixed point of the MRF. This is the second main observation we make in this paper. The modified Ricci flow does seem to have some connection with geometric entropy: 
its fixed points are extrema of the Bekenstein-Hawking entropy. Note that the function $f$ is positive in the Schwarzschild exterior and that it degenerates to zero on the inner boundary $r=2 M$. The form of $f$ motivates the identification of the pair $\left(h_{a b}, f\right)$ with the static spacetime

$$
d s^{2}=-f(x)^{2} d t^{2}+h_{a b} d x^{a} d x^{b}
$$

This gives a Lorentzian spacetime interpretation of $f$ as the lapse function or the redshift factor.

\section{GENERALISATION TO ADS}

For the compact spatial topologies studied by Perelman, there was room to modify the RHS of the Ricci flow by the addition of a "cosmological constant" $\lambda h_{a b}$. This led to the possibility of additional Ricci solitons, shrinking and expanding breathers. In the last two sections, we required that our metric $h_{a b}$ tend to a fixed flat metric at infinity and so $\lambda$ had to vanish to preserve the asymptotic conditions. It is interesting to ask what happens if we replace asymptotic flatness by asymptotically AdS. The arguments of the last two sections go through with appropriate slight modifications. In the equations (419), one simply replaces the Ricci tensor $R_{a b}$ by $R_{a b}-\lambda h_{a b}$, where $\lambda$ is a negative cosmological constant. Correspondingly, (11) acquires an extra term $\lambda r a a^{\prime}$ on the right hand side. As before we see that the AdS Schwarzschild space, which has $a(r)=\left(1-2 M / r-\lambda r^{2} / 3\right)^{-1}$ does not satisfy the soliton equation.

However, we modify the flow to read

$$
\frac{\partial h_{a b}}{\partial \tau}=-2 f\left(R_{a b}-l a m b d a h_{a b}\right)+2 D_{a} D_{b} f
$$

supplemented by an evolution equation for $f$ :

$$
\frac{\partial f}{\partial \tau}=D^{2} f+\lambda f
$$

Then we find that the AdS Schwarzschild is indeed a Ricci Soliton of the modified flow with $f$ given by $f=\left(1-2 M / r-\lambda / 3 r^{2}\right)^{1 / 2}$. Thus our main conclusions generalise easily to include AdS space. 


\section{CONCLUSION}

In his paper[3], Perelman remarks that the interplay between statistical physics and (Lorentzian) geometry occurs in the subject of black hole thermodynamics. He appears to suggest possible connections between his entropy functional and black hole thermodynamics. Perelman was working with compact Riemannian manifolds. It seems clear that for a discussion of geometric entropy we need to have non-compact manifolds, so that we can define a region of no escape. The main result of this paper is the observation that the Perelman entropy is not the geometric entropy, at least if one uses the natural identications we have made between initial data and spacetimes.

Another observation we make is that a small modification of Pereman's flow does seem to relate to geometric entropy. It would seem important to understand the properties of the new flow and possibly relate it to the Perelman flow. For instance, What can one say about the long term behaviour of both flows starting with an arbitrary initial positive curvature metric in the asymptotically flat context. How does one choose the initial $f$ ? Does the MRF admit a gradient formulation? We are presently studying both flows in the linear regime in the neighborhood of their Ricci solitons.

Several papers have appeared [18, 19, 20, 21] in the physics literature about physical applications of the Ricci flow. Many of them are motivated by the renormalisation group, string theory or conformal field theory. Our motivation is from the viewpoint of classical general relativity. In GR, there are a number of results of a purely differential geometric character which also have a strong thermodynamic flavour. Examples abound in black hole physics, but let us mention two: the Penrose inequality and the Hawking area theorem. These results do not appeal to Einstein's equations but only to energy conditions. The Ricci flow techniques of Perelman do seem to be a natural tool to attack such problems.

[1] D. Friedan 1980 Phys. Rev. Lett. 45, 1057

[2] R. S. Hamilton 1982 Three-manifolds with positive Ricci curvature J. Differential Geom. 17 255-306

[3] Grisha Perelman 2002 The entropy formula for the Ricci flow and its geometric applications Preprint math.DG/0211159 
[4] Huai-Dong Cao and Bennett Chow 1999 Recent developments on the Ricci flow Bull. Amer. Math. Soc. 36 59-74

[5] R.M. Wald 1984 General Relativity University of Chicago Press

[6] Peter Topping 2006 London Mathematical Society Lecture Notes Series Cambridge University Press

[7] Richard Schoen and Shing-Tung Yau 1979 Positivity of the Total Mass of a General SpaceTime Phys. Rev. Lett. 43 1457-1459

[8] Edward Witten 1981 A New Proof of the Positive Energy Theorem Commun. Math. Phys. 80 $381-402$

[9] R.Penrose, R.D Sorkin and E.Woolgar A Positive Mass Theorem Based on the Focusing and Retardation of Null Geodesics 1993 Preprint gr-qc/9301015

[10] Roger Penrose 1973 Ann. NY Acad. of Science 224

[11] Robert Geroch 1973 Ann. NY Acad. of Science 225

[12] P.S. Jang and R.M. Wald 1977 J. Math. Phys. 1841

[13] Gerhard Huisken and Tom Ilmanen 2001 J. Differential Geom. 59 353-437

[14] Hubert L.Bray 2003 Preprint math.DG/0304261, Proceedings of the ICM, Beijing 2002 2 257-272

[15] Hubert L.Bray 2001 J. Diff. Geom. 59 177-267

[16] R.D. Sorkin and M. Varadarajan 1996 Class. Quant. Grav. 131949

[17] We are suppressing a subtlety here. Quantum effects lead to black hole evaporation and a further increase of entropy. This paper is concerned only with classical GR.

[18] Ioannis Bakas 2005 textitComptes Rendus Physique 6 175-184, Preprint hep-th/0702034

[19] T. Oliynyk, V. Suneeta and E. Woolgar 2006 Nucl. Phys. B739 441

[20] Matthew Headrick and Toby Wiseman, Class. Quantum Grav. 23 (2006), 6683.

[21] T. Oliynyk and E. Woolgar 2006 Preprint math-dg/0607438

[22] The term "modified Ricci flow" is sometimes used in the mathematical literature to describe Ricci flow with surgery. This is not to be confused with the flow described here. 